\title{
CYCLIC THERMAL CREEP MODEL FOR THE BODIES OF REVOLUTION
}

\author{
D. V. Breslavs'kyi, Yu. M. Korytko, \\ and O. K. Morachkovs'kyi
}

UDC 539.3

\begin{abstract}
The new method of solving thermal creep problems, accounting for cyclic variations of external force and thermal fields was put forward. Asymptotic expansions and period averagings were used to derive the systems of basic and auxiliary equations. The equation of state for cyclic thermal creep was proposed and validated. Numerical simulation of the creep of cylinders under cyclic temperature variations was carried out.
\end{abstract}

Keywords: cyclic thermal creep, creep rupture strength, damage, cyclic loading, temperature, bodies of revolution.

Introduction. One of the most widespread classes of engineering structural elements is the bodies of revolution, viz pressure vessels and valves, pipe lines, internal combustion engine cylinders, steam and gas turbine rotors, etc. Since such structural elements operate under the joint action of force and thermal fields, evaluation of their deformation behavior is one of the important problems for ensuring necessary service life. As is known, the level and frequency of load and temperature variations can greatly influence creep and time to fracture [1-7].

Increasing requirements to materials consumption, life, and reliability gave rise to significant results obtained in creep and creep rupture strength calculations for materials and structures [1, 5-7]. Polymer structural elements are usually subjected to analysis for studying the interaction between cyclic force and thermal fields [8]. Most calculations of service life and creep rupture strength of structural elements manufactured from metals and their alloys are performed only for the case of time-invariant thermal fields. The exception is represented by the studies under the direction of Academician G. S. Pisarenko [6, 7, 9, 10], in which equations of state for materials on cyclic high-temperature heating were suggested and verified as well as by experimental investigations of the models of high-temperature structural elements (blades, internal combustion engine pistons, etc.).

Thus, the development of a method for calculating cyclic thermal creep and damage accumulation in structural elements remains the topical problem of modern mechanics. The new equations of state are put forward to describe the creep of materials at constant temperature, high- and low-rate stress variations [11, 12]. However, for the practically significant case of cyclic temperature variations, with creep developing in structural materials, the equations of state suitable for calculations in the multiaxial stress state heretofore have not been derived.

In this study, the statement of the problem is presented and the new equations of state are proposed to describe the cyclic thermal creep of metallic materials and their alloys. The calculations are based on the methods of two time scales and asymptotic expansions. Calculated and experimental cyclic creep data obtained for different temperatures and thermal cycles were compared and verified. The problem was solved by the finite element method and by multistep time integration. The method of solving cyclic thermal creep problems was applied to the two-dimensional ones. As an example, the solutions of cyclic thermal creep problems for cylinders were presented and new results as regards the stress distribution and stress redistribution termination time were obtained.

Statement of the Problem. Consider the body of the volume $V$, secured on the part of the surface $S_{1}$, which is loaded with the surface forces $p_{i}$ on the part of the surface $S_{2}$ and the volume forces $f_{i}$. External volume forces are assumed to be time-invariant, $f=f(x), x \in V$, and surface forces in the general case possess constant and cycle-variable components. As a rule, they are represented as the Fourier series

National Technical University "Kharkiv Polytechnic Institute," Kharkiv, Ukraine. Translated from Problemy Prochnosti, No. 2, pp. 33 - 46, March - April, 2011. Original article submitted September 10, 2009. 


$$
p_{i}=p_{i}^{0}+\Phi_{i}(t), \quad x \in S_{2}
$$

Introduce commonly accepted designations for the displacement vector $u$, the displacement rate $\dot{u}$, the stress $\left(\sigma_{i j}=\sigma_{j i}\right)$ and strain $\left(e_{i j}=e_{j i}\right)$ tensors, which are the functions of the $x_{i}(i=1,2,3)$ coordinates and time $t$. The strain tensor found by thermal expansion is denoted by $e_{i j}^{T}$. Irreversible creep strains without account of their effect on compressibility are represented by the tensor $c_{i j}=c_{j i}$.

Considering the above assumptions and temperature-time variations, the solution of the nonstationary problem of heat transfer is obtained. The initial-boundary problem of the body creep is formulated as the system of equations

$$
\begin{aligned}
& \left\{\begin{array}{l}
\varepsilon_{i j}=e_{i j}+c_{i j}+e_{i j}^{T}, \quad \sigma_{i j, j}+f_{i}=\rho \frac{\partial^{2} u_{i}}{\partial t^{2}}, \\
\varepsilon_{i j}=\frac{1}{2}\left(u_{i, j}+u_{j, i}\right), \quad x_{i} \in V, \\
\sigma_{i j}=D_{i j k l}\left(\varepsilon_{k l}-c_{k l}-e_{k l}^{T}\right), \quad \sigma_{i j} n_{j}=p_{i}^{0}+\Phi_{i}(t), \quad x_{i} \in S_{2},
\end{array}\right. \\
& \left.u_{i}\right|_{S_{1}}=\bar{u}_{i}, \quad u_{i}(x, 0)=c_{i j}(x, 0)=0, \quad e_{i j}^{T}=\alpha_{i j}\left(\varphi(T)-\varphi\left(T_{0}\right)\right), \\
& a^{2} \Delta \varphi+Q=\dot{\varphi}, \quad a^{2}=\frac{k}{c \rho}, \quad Q=\frac{F}{c \rho},\left.\quad \varphi(t)\right|_{S_{3}}=\bar{\varphi}(t),
\end{aligned}
$$

where $n$ is the unit outer normal to the body boundary, $j=1,2,3, D_{i j k l}$ is the tensor of elastic material properties, $\bar{u}_{i}$ is the known time-invariant displacements of the points of the surface $S_{1}, \varphi=\varphi(T)$ is the temperature distribution function, $\alpha_{i j}$ is the coefficients of thermal expansion, $Q$ is the thermal flow density function, $k$ is the heat transfer coefficient, $c$ is the thermal conductivity coefficient of the material, and $\rho$ is the mass density.

The equations of state based on the Bailey-Norton and Rabotnov-Kachanov equations and the exponential creep strain rate-temperature dependence [5] are given as

$$
\begin{gathered}
\dot{c}_{i j}=\frac{3}{2} \frac{B\left(\sigma_{i}\right)^{n-1}}{(1-\omega)^{l}} S_{i j} \exp \left(-\frac{H}{R \varphi(T)}\right), \\
\dot{\omega}=\frac{D \sigma_{e}^{m}}{(1-\omega)^{l}}, \quad \omega(0)=\omega_{0}, \quad \omega\left(t_{*}\right)=1,
\end{gathered}
$$

where $B, D, n, m$, and $l$ are the material constants, determined from experimental creep and creep rupture strength curves over a preset temperature range, $R$ is the absolute gas constant, $\omega$ is the damage parameter, and $H$ is the creep activation energy.

Solution Method. Assume that the external volume forces are time-invariant, $f=f(x), x \in V$ and the surface forces contain time-invariant $\left[p_{i}^{0}=p_{i}^{0}(x)\right]$ and cycle-variable $\left[\Phi_{i}(t)\right]$ loading components $p_{i}=p_{i}^{0}+\Phi_{i}(t)$, $x \in S_{2}$.

The cycle-variable components of the surface forces on $S_{2}$ are taken as the sum

$$
\Phi_{i}(t)=p_{i}^{\max } \Phi^{1}(t)+p_{i}^{a} \Phi^{(2)}(t)
$$

corresponding to the cyclic body loading that varies during the time $T$ and approximated by the Fourier series with the coefficients $a_{k}$ and $b_{k}$ 


$$
\begin{gathered}
\Phi^{1}(t)=\sum_{k=1}^{\infty} \Phi_{k}=\sum_{k=1}^{\infty} A_{k} \sin \left(\Omega_{k} t+\beta_{k}\right), \\
\Phi_{k}(t)=a_{k} \cos \left(\frac{2 \pi k}{T} t\right)+b_{k} \sin \left(\frac{2 \pi k}{T} t\right)=A_{k} \sin \left(\Omega_{k} t+\beta_{k}\right), \\
A_{k}=\sqrt{a_{k}^{2}+b_{k}^{2}}, \quad \Omega_{k}=\frac{2 \pi k}{T}, \quad \beta_{k}=\arctan \left(a_{k} / b_{k}\right),
\end{gathered}
$$

where $p_{i}^{\max }$ is the amplitudes of corresponding surface loading components.

System (2) is essentially nonlinear. It stems from the fact that the irreversible strain tensor components $c_{i j}$ are nonlinearly related to the stress tensor components.

Incommensurability of the static and cyclic periods allows asymptotic expansions in terms of the small parameter $\mu$ to be written for the unknowns of system (2) [13]

$$
z=z^{0}(x, t)+\sum_{i=1}^{\infty} \mu^{i} z^{i}(x, t, \xi)
$$

where $z$ stands for any unknown of system (2), and $x, t$, and $\xi$ are formally independent variables.

Since $z^{i}$ is the periodic function of $\xi$, its average-per-period values are equal to zero

$$
\left\langle z^{i}(x, t, \xi)\right\rangle=\int_{0}^{1} z^{i} d \xi=0 .
$$

As a rule, for the time derivative we have

$$
\frac{d}{d t} z^{i}=\frac{\partial}{\partial t} z^{i}+\frac{1}{\mu} \frac{\partial}{\partial \xi} z^{i}
$$

Then restrict to the first approximation of asymptotic expansions (8)

$$
z \cong z^{0}(t)+\mu z^{1}(\xi)
$$

Notice that in equalities (11) the functions on the coefficients $\mu$ are dependent not only on $\xi$ but also on the spatial $x$ coordinates.

In accordance with the accepted asymptotic approximation with the account of (11), we have

$$
\begin{array}{rlrl}
u_{i} & \cong u_{i}^{0}(t)+\mu u_{i}^{1}(\xi), & v_{i} \cong v_{i}^{0}(t)+\mu v_{i}^{1}(\xi), \\
v_{i}^{1}=1 / \mu u_{i, \xi}^{1}, & v_{i, \xi}^{1}=1 / \mu^{2} u_{i, \xi \xi}^{1}, \\
\sigma_{i j} \cong \sigma_{i j}^{0}(t)+\mu \sigma_{i j}^{1}(\xi), & \varepsilon_{i j} \cong \varepsilon_{i j}^{0}+\mu \varepsilon_{i j}^{1}, \\
\varepsilon_{i j}^{0}=\frac{1}{2}\left(u_{i, j}^{0}+u_{j, i}^{0}\right), & \varepsilon_{i j}^{1}=\frac{1}{2}\left(u_{i, j}^{1}+u_{j, i}^{1}\right) .
\end{array}
$$

Substitute expansions (12) and (13) into system of equations (2) and solve the relations using period averaging (9). Then for the equilibrium equations and for the stress-strain relations we accordingly have 


$$
\begin{gathered}
\left\langle\sigma_{i j, j}^{0}\right\rangle+\left\langle\mu \sigma_{i j, j}^{1}\right\rangle+\left\langle f_{i}^{0}\right\rangle+\left\langle\mu f_{i}^{1}\right\rangle=0 \\
\left\langle\varepsilon_{i j}^{0}\right\rangle+\left\langle\mu \varepsilon_{i j}^{1}\right\rangle=\left\langle C_{i j k l} \sigma_{k l}^{0}\right\rangle+\left\langle\mu C_{i j k l} \sigma_{k l}^{1}\right\rangle+\left\langle c_{i j}^{0}\right\rangle+\left\langle\mu c_{i j}^{1}\right\rangle .
\end{gathered}
$$

Average coefficients corresponding to slow-drift motion are equal to those values, $\left\langle z^{i}\right\rangle=0$ according to (9).

Asymptotic expansions and period averagings allow initial system (2) to be divided into the two subones. The first subsystem results from the relations remaining after averaging and describes slow-drift motion

$$
\left\{\begin{array}{l}
\sigma_{i j, j}^{0}+f_{i}^{0}=0, \quad x_{i} \in V, \\
\sigma_{i j}^{0} n_{j}=p_{i}^{0}, \quad x_{i} \in S_{2}, \\
\varepsilon_{i j}^{0}=C_{i j k l} \sigma_{k l}^{0}+c_{i j}^{0}, \quad \varepsilon_{i j}^{0}=\frac{1}{2}\left(u_{i, j}^{0}+u_{j, i}^{0}\right), \quad x_{i} \in V, \\
\left.u_{i}^{0}\right|_{S_{1}}=\bar{u}_{i}, \quad u_{i}^{0}\left(x_{i}, 0\right)=0, \quad c_{i j}^{0}=0 .
\end{array}\right.
$$

Here all the unknowns are the functions, slowly changing on the time scale $t$. Inertial forces can be neglected because of slow changes in external loads.

The second subsystem describing fast-drift motion is obtained by subtracting subsystem (16) from system (2)

$$
\left\{\begin{array}{l}
\sigma_{i j, j}^{1}=\frac{\rho}{\mu^{2}} \frac{\partial^{2} u_{i}^{1}}{\partial \xi^{2}}, \quad x_{i} \in V, \\
\sigma_{i j}^{1} n_{j}=\frac{1}{\mu}\left(p_{i}^{\max } \Phi^{1}(t)\right), \quad x_{i} \in S_{2}, \\
\varepsilon_{i j}^{1}=\frac{1}{2}\left(u_{i, j}^{1}+u_{j, i}^{1}\right)=C_{i j k l} \sigma_{k l}^{1}, \quad x_{i} \in V, \\
\left.u_{i}^{1}\right|_{S_{1}}=0, \quad u_{i}^{1}(x, 0)=0, \quad c_{i j}^{1}=0, \quad 0<\xi<1 .
\end{array}\right.
$$

At low frequencies of the body motion, the inertial forces in (17) can be neglected.

Subsystems (16) and (17) that describe slow- and fast-drift motions are not independent. They are related by the equation of state [12]. In the examined case, it is the equation of cyclic thermal creep.

Equation of State. The equations of state for cyclic creep of structural materials were used to calculate creep and damage accumulation in the multiaxial stress state [10-12].

The cyclic loading $\sigma=\sigma^{0}+\sigma^{1}$ is examined under the combined constant stress $\sigma^{0}$ and stress $\sigma^{1}$ varying with the cyclic frequency $f_{1}=1 / T$ during the operating cycle $T\left(f_{1}<<1 \mathrm{~Hz}\right)$. The stress $\sigma^{1}$ is represented by the periodical Fourier series, and cyclic stress variations for the above loading conditions are written as

$$
\sigma=\sigma^{0}+\sigma^{1}=\sigma^{0}\left(1+\sum_{k=1}^{\infty} M_{k} \sin \left(\frac{2 \pi k}{T} t+\beta_{k}\right)\right),
$$

where $M_{k}=\sigma^{a k} / \sigma^{0}$ is the amplitude factors in cyclic creep, $\sigma^{0} \neq 0$, and $\beta_{k}$ is the phase.

Creep strain rates can vary as a result of stress-time and temperature-time variations. The temperature function is also represented by the Fourier series, and the temperature variation cycle asymmetry parameter $M_{l}^{T}=\varphi^{a l} / \varphi^{0}$ is 
introduced as the ratio of amplitude temperature component to its minimum value over a thermal variation period. Then the cyclic temperature variations will have the form

$$
\varphi=\varphi^{0}+\varphi^{1}=\varphi^{0}\left(1+\sum_{l=1}^{\infty} M_{l}^{T} \sin \left(\frac{2 \pi l}{T_{\varphi}} t+\beta_{l}^{T}\right)\right) .
$$

The creep of the specimen cut out from a metallic material would be studied within general kinetic dependences of the theory of structural parameters proposed by Rabotnov. Specify the equation of state for creep in the uniaxial stress state, taking the Bailey-Norton and Rabotnov-Kachanov equations and the most-used exponential temperature dependence for creep and damage accumulation [6]

$$
\dot{c}=B \frac{(\sigma)^{n}}{(1-\omega)^{k}} \exp \left(\frac{-H}{R \varphi}\right), \quad \dot{\omega}=D \frac{(\sigma)^{r}}{(1-\omega)^{l}} \exp \left(\frac{-H}{R \varphi}\right), \quad \omega(0)=\omega_{0}, \quad \omega\left(t_{*}\right)=\omega_{*},
$$

where $c(t)$ is the irreversible creep strains, $\omega(t)$ is the damage parameter, $r$ and $k$ are the material constants determined from experimental creep and creep rupture strength curves, and $\omega_{*}$ is the damage parameter at the latent fracture stage termination time $t_{*}$.

Incommensurability of the static and cyclic loading periods allows asymptotic expansions and period averaging to be used for describing cyclic creep and damage accumulation.

Constant-temperature deformation is first examined. Assume that the total period of creep $t_{*}$ exceeds the period $T$ of the cyclic stress $\sigma^{1}$, and introduce the small parameter $\mu=T / t_{*}<<1$ and the two time scales: $t$ for slow creep under cyclic loading and $\xi=t / T$ for $T$-period variable fast creep under stress, $0 \leq \xi \leq 1$.

The solution would be sought as the asymptotic small-parameter expansion

$$
\begin{gathered}
c \cong c^{0}(t)+\mu c^{1}(\xi), \\
\omega \cong \omega^{0}(t)+\mu \omega^{1}(\xi),
\end{gathered}
$$

where $c^{0}(t), \omega^{0}(t), c^{1}(\xi)$, and $\omega^{1}(\xi)$ are the functions that correspond to creep and damage on the slow $(t)$ and fast

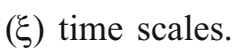

Since creep and damage are only slow-time dependent, after $\xi$-period averaging with the account of

$$
\left\langle c^{1}(\xi)\right\rangle=\int_{0}^{1} c^{1}(\xi) d \xi \cong 0, \quad\left\langle\omega^{1}(\xi)\right\rangle=\int_{0}^{1} \omega^{1}(\xi) d \xi \cong 0
$$

the time $\xi$ can be excluded from expansions (21) and (22).

Using asymptotic expansions and (21) and (22) for equations (20), after $T$-period averaging under cyclic stress, we have [12]

$$
\begin{gathered}
\dot{c}=B g_{n} \frac{\left(\sigma^{0}\right)^{n}}{(1-\omega)^{k}}, \quad g_{n}=\int_{0}^{1}\left(1+\sum_{k=1}^{\infty} M_{k} \sin (2 \pi k \xi)\right)^{n} d \xi, \\
\dot{\omega}=D g_{r} \frac{\left(\sigma^{0}\right)^{r}}{(1-\omega)^{l}}, \quad \omega(0)=\omega_{0}, \quad \omega\left(t_{*}\right)=\omega_{*}, \quad g_{r}=\int_{0}^{1}\left(1+\sum_{k=1}^{\infty} M_{k} \sin (2 \pi k \xi)\right)^{r} d \xi,
\end{gathered}
$$

where $g_{n}$ and $g_{r}$ are the functions of cyclic loading amplitude factors. 
Relations (24) and (25) are taken as the equations of state for cyclic creep under the combined static and slow-variable stresses: $\sigma=\sigma^{0}+\sigma^{1}$.

If the specimen temperature is time-variable, then similarly to the above procedure, we derive the equations of state for the case of cyclic temperature variations

$$
\begin{gathered}
\dot{c}=B g_{n}^{T} \frac{\left(\sigma^{0}\right)^{n}}{(1-\omega)^{k}}, \quad g_{n}^{T}=\int_{0}^{1} \exp \left\{-Q\left[\varphi^{0}\left(1+\sum_{l=1}^{\infty} M_{l}^{T} \sin (2 \pi l \xi)\right]\right]^{-1}\right\} d \xi, \\
\dot{\omega}=D g_{r}^{T} \frac{\left(\sigma^{0}\right)^{r}}{(1-\omega)^{l}}, \quad g_{r}^{T}=\int_{0}^{1} \exp \left\{-Q\left[\varphi^{0}\left(1+\sum_{l=1}^{\infty} M_{l}^{T} \sin (2 \pi l \xi)\right)\right]^{-1}\right\} d \xi, \\
\omega(0)=\omega_{0}, \quad \omega\left(t_{*}\right)=\omega_{*},
\end{gathered}
$$

where $g_{n}^{T}$ and $g_{r}^{T}$ are the functions of cyclic heating-cooling amplitude factors.

Examine the more complicated case of joint cyclic temperature and mechanical loading effects. Upon the application of asymptotic expansions and temperature cycle period averaging to relations (24) and (25), we obtain the new cyclic thermal creep equations

$$
\dot{c}=B g_{n} g_{n}^{T} \frac{(\sigma)^{n}}{(1-\omega)^{k}}, \quad \dot{\omega}=D g_{r} g_{r}^{T} \frac{(\sigma)^{r}}{(1-\omega)^{l}}, \quad \omega(0)=\omega_{0}, \quad \omega\left(t_{*}\right)=\omega_{*}
$$

Proposed equations of state for cyclic thermal creep with polyharmonic loading within the cycle (28) account for the effect of polyharmonic stress amplitude factors and cyclic temperature variations on creep strains and the damage parameter. The equations of state provide the mathematical representation of the nonlinear deformation of the material under thermomechanical static and cyclic loadings.

Stress-strain rates in the uniaxial stress state corresponding to equivalent stresses in the multiaxial one, postulated by the classical creep theory, are introduced into equations of state (28) to be generalized for the case of the multiaxial stress state. The Mises stress intensity can be taken as equivalent stresses for cyclic creep and dynamic loading, which is substantiated by experimental data obtained in the multiaxial stress state [14], etc., while in the kinetic equations for the damage parameter that correspond to creep rupture strength curves, the equivalent stresses are consistent with the Pisarenko-Lebedev creep rupture strength criterion

$$
\begin{gathered}
\dot{c}=B g_{n} g_{n}^{T} \frac{3\left(\sigma_{i}\right)^{n-1}}{2(1-\omega)^{k}} S_{i j}, \\
\dot{\omega}=D g_{r} g_{r}^{T} \frac{\left(\sigma_{e q}\right)^{r}}{(1-\omega)^{l}}, \quad \omega(0)=\omega_{0}, \quad \omega\left(t_{*}\right)=1, \\
\sigma_{i}=\sqrt{\frac{2}{3} S_{i j} S_{i j}}, \quad \sigma_{e q}=\alpha \sigma_{1}+(1-\alpha) \sigma_{i},
\end{gathered}
$$

where $\sigma_{i}$ is the stress intensity, $\sigma_{e q}$ is the equivalent stress, $\sigma_{1}$ is the maximum normal stress, $S_{i j}$ is the stress tensor deviator $\left(\sigma_{i j}\right)$ components, and $\alpha$ is the parameter of material sensitivity to a fracture mode ( $\alpha=1$ for brittle, $\alpha=0$ for plastic, and $0<\alpha<1$ for mixed ones). 

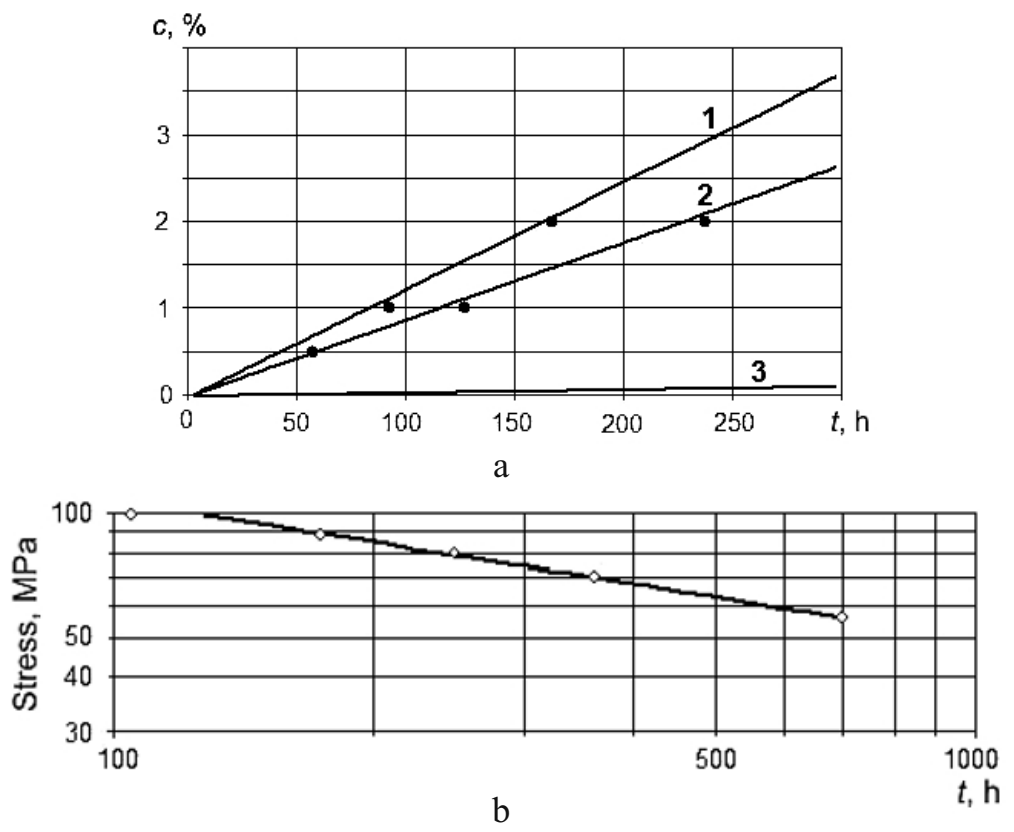

Fig. 1. Static (1,3) and cyclic (2) creep curves (a) and creep rupture strength curve (b): (1) $T=1003 \mathrm{~K}$, (3) $T=923 \mathrm{~K}$ (points correspond to experimental data).

The derived equations of state for cyclic creep and damage accumulation were validated by approving equations (28) with experimental data obtained by several authors. The behavior of AMg6, N 155, and S 321 alloys, RC-130-A and RS50 titanium, Inconel X, and others was investigated over the operating temperature range with different stress levels under static and variable thermomechanical loadings.

As an example, known experimental data [15] are compared with the data obtained by the derived equations of state. Static and cyclic creep curves for 321 steel specimens and results of creep rupture strength analysis for this steel at cycle-variable temperature are presented in Fig. 1. Trapezoid-cycle temperature variations from 293 to $1003 \mathrm{~K}$ with a 2 -h period and 1.5-h holding time at maximum temperature are represented by curve 2 .

Calculated and experimental data are in rather good agreement.

Experimental verification of the derived equations demonstrated a fairly high level of their adequacy, thus, they can be recommended for analysis of the creep rupture strength of engineering structural elements. A maximum error in description of cyclic creep and creep rupture strength curves makes up 5-10\%.

Cyclic Thermal Creep of a Cylinder. Simulation of creep and damage accumulation under cyclic temperature and external load variations is based on finite element algorithms for the boundary problem and difference ones for solving the initial problem. For calculations use the element in the form of a triangular cross-section body of revolution. The software is described in $[13,16]$.

The results of solving the cyclic thermal creep problem are given for a long cylindrical thick-walled tube with $0.07-\mathrm{m}$ inner and $0.1-\mathrm{m}$ outer radii. The tube is made of carbon steel with the following physicomechanical properties: $E=(1.7-1.9) \cdot 10^{5} \mathrm{MPa}, v=0.3$, and $a=(1.3-1.4) \cdot 10^{-5} \mathrm{~K}^{-1}$ over the temperature range of 573-773 K. The creep constants for (29) were determined from the data of the Tapsell classical experiments [17]: $B=$ $4.27 \cdot 10^{-18} \mathrm{MPa}^{-n} / \mathrm{s}, n=6.42, Q=9.8 \cdot 10^{3} \mathrm{~K}$, and $A=3.3 \cdot 10^{5}$. The uniform mesh with 2000 finite elements and 1111 modes was used.

First examine the creep of a uniformly heated cylinder under cyclic temperature variations. Assume that the temperature of the tube varies cyclically by a 12-min rectangular cycle. During the first half-cycle (6 min) the cylinder is uniformly heated up to $773 \mathrm{~K}$, during the second half-cycle it is within a 573-K uniform temperature field. Moreover, it is assumed that the cylinder is heated and cooled instantaneously. The tube is under a 20-MPa internal pressure. 

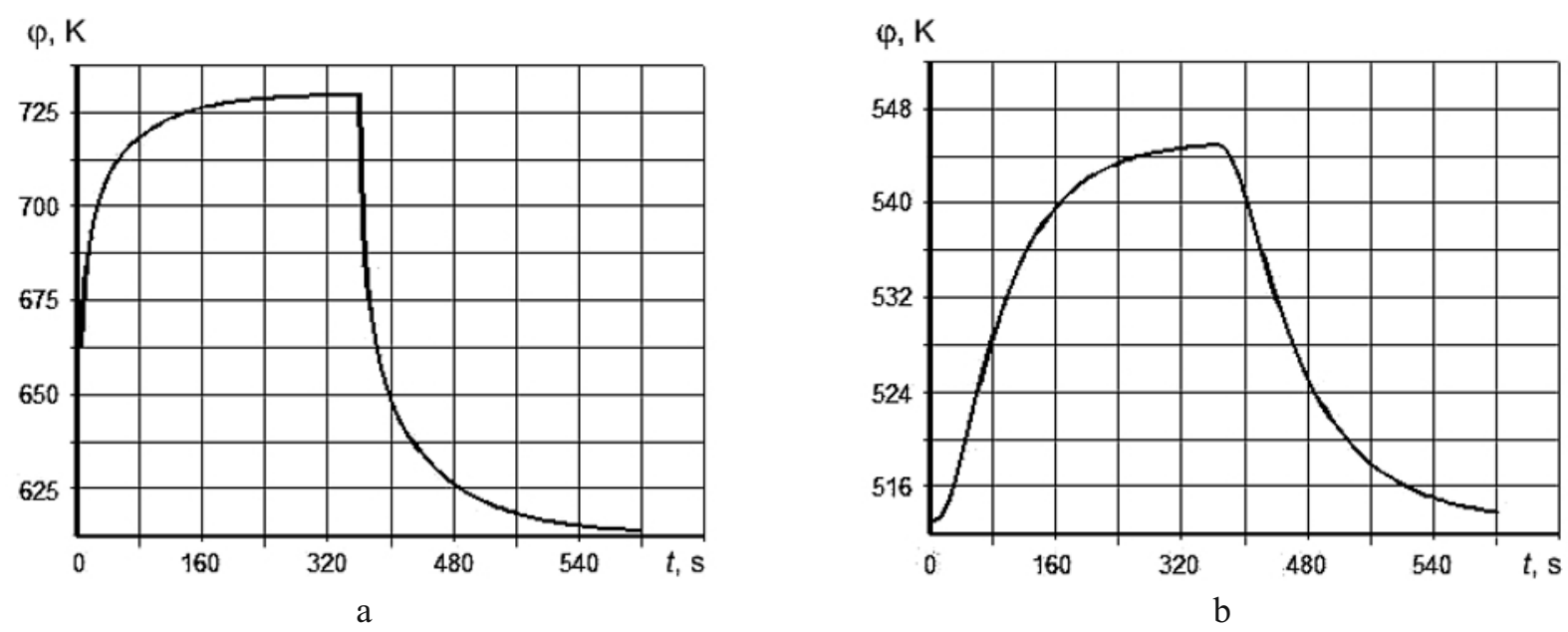

Fig. 2. Cyclic temperature-time variations for inner (a) and outer (b) radii points.

As the results demonstrated, the stress redistribution termination time makes up $600 \mathrm{~h}$. For the constant temperature of $573 \mathrm{~K}$, the time of stress redistribution is $25,000 \mathrm{~h}$, for $773 \mathrm{~K}$ it is $300 \mathrm{~h}$. Notice a quite significant time reduction of stress redistribution (more than 40 times) in cyclic creep as compared to static one at $573 \mathrm{~K}$ and twofold creep slowing-down as compared to creep at $773 \mathrm{~K}$. The stress distribution in this case is qualitatively identical to that in static creep for the cylinder under internal pressure (e.g., [6]).

The analysis points to an essential effect of cyclic temperature variations in the cylinder under internal pressure on the stress redistribution termination time. At cyclic temperature variations in the case of instantaneous heating, the stress redistribution pattern does not change but the time of their redistribution changes considerably.

Now examine the cyclic thermal creep of the carbon steel cylinder at nonuniformly distributed temperature over its cross-section. Assume that the gas flow temperature inside the tube varies cyclically with a 12-min period. During first $6 \mathrm{~min}$ it is $753 \mathrm{~K}$, during the second half-cycle it is $613 \mathrm{~K}$. The environmental temperature on its outer surface remains constant and makes up $613 \mathrm{~K}$. The cylinder is under a 5-MPa internal pressure.

Analyze thermal conditions in the tube. For this, it is necessary to solve the nonstationary problem of convective heat transfer. The numerical solution yields the temperature distribution along the cylinder radius for different times. The solution shows that temperature varies continuously within the examined cycle, which is intrinsic to continuous stress variations. Temperature-time curves for inner and outer cylinder radii points are shown in Fig. 2.

The results of solving the above problem are taken as the initial data for the solution of the set of thermoelasticity equations. Temperature distributions over the cylinder at different times $(30,60,90$, and $120 \mathrm{~s}$, etc. with a 30-s step) were used.

Then cyclic stress tensor component and stress intensity variations were interpolated for each discretization point. Figure 3 illustrates cyclic stress intensity-time variations.

Temperature-time and stress-time dependences in different points of the radius were employed for establishing stress and temperature cycle asymmetry functions. The calculations with these functions demonstrate that cyclic temperature variations lead to a considerable change in the stress redistribution termination time: from $80 \mathrm{~h}$ in the constant radius-nonuniform temperature field to $30 \mathrm{~h}$ in the case of creep under cyclic temperature variations. Circumferential and radial stress distribution curves at $t=30 \mathrm{~h}$ are shown in Fig. 4.

As is seen, the stress redistribution pattern changes considerably, which is explained by a lower creep strain rate in the central areas of the cylinder.

Examine the results of solving the same but simplified problem: it is assumed that instantaneous heating follows a rectangular temperature variation cycle, which provides a rectangular pattern of thermal stress variations. The temperature distribution along the radius is taken in accordance with the distribution typical of an average cycle temperature. 

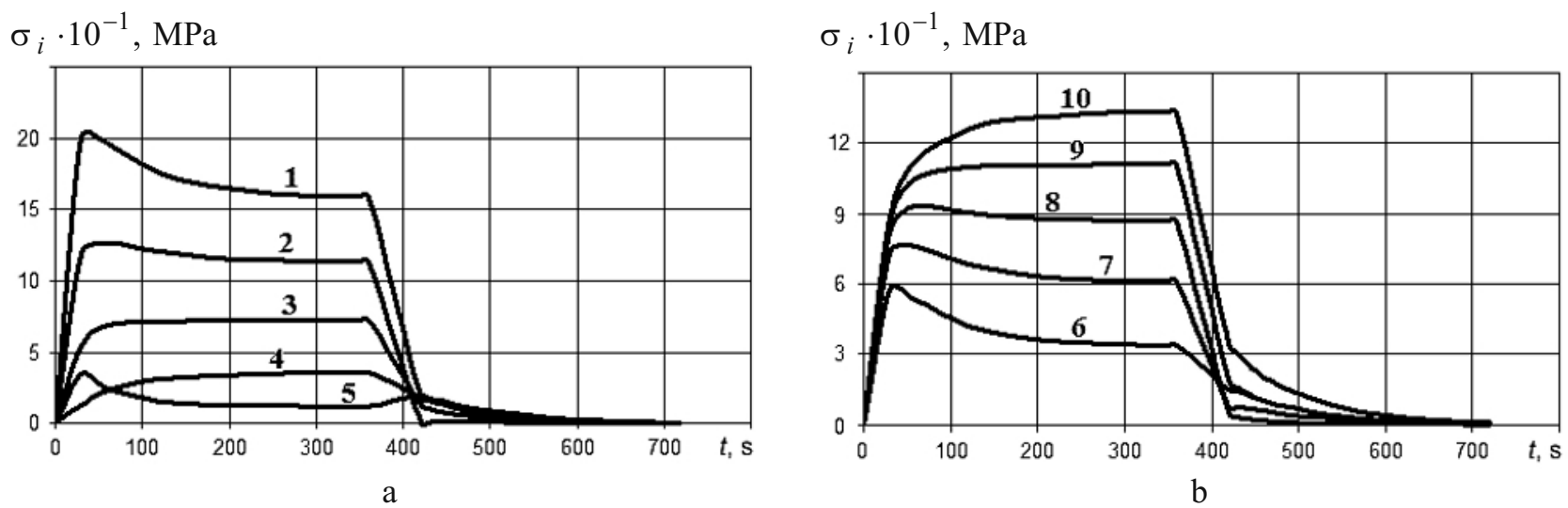

Fig. 3. Stress intensity-time variations within a cycle: points whose coordinates change with a 3-mm step from the inner surface to the center of the cylinder (1-5) and from the center of the cylinder to its outer surface $(6-10)$.

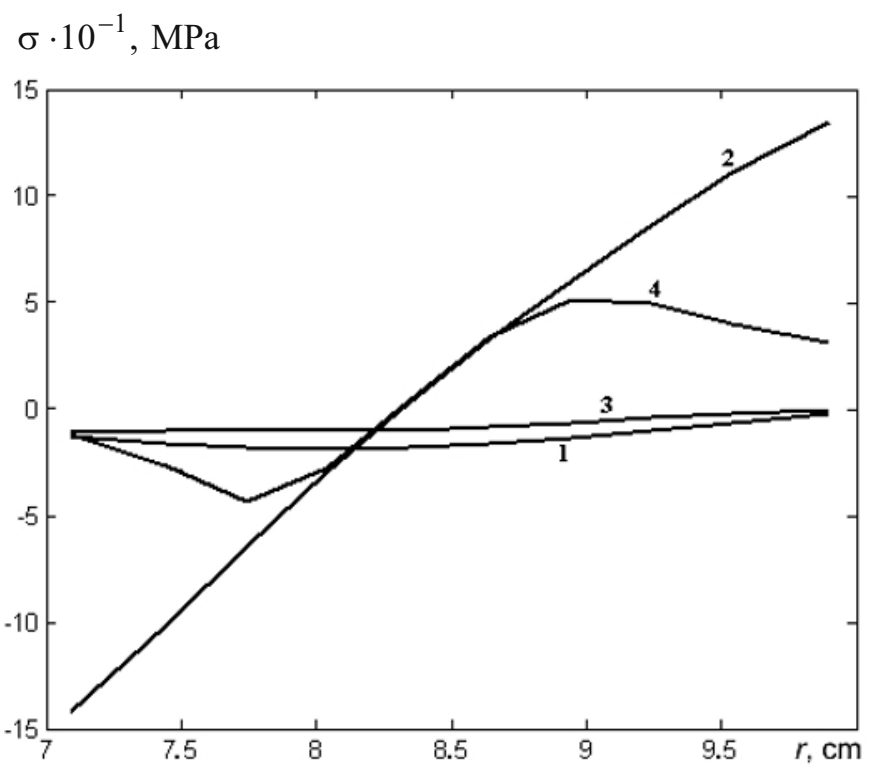

Fig. 4. Radial $(1,3)$ and circumferential $(2,4)$ stress distributions: $(1,2) t=0 ;(3,4) t=30 \mathrm{~h}$.

The results are slightly different from those cited in Fig. 4. The stress redistribution termination time is $30-40 \mathrm{~h}$. This points to the fact that the account of gradual heating and cooling in the cycle does not change greatly the stress redistribution pattern in creep or influence essentially its termination time.

Another calculation was performed to estimate the contribution of the $g_{2}(\varphi)$ function to the obtained solution. The temperature dependence of creep constants for the steel within a (643-723)-K range was neglected, while the calculations made use of their values obtained for an average temperature of $683 \mathrm{~K}$.

The results of this calculation did not also reveal any essential effect of the temperature dependence of the constants in (29) on the stress redistribution pattern and their redistribution termination time. Small differences as compared to the refined solution could be found only in the cylinder areas near its outer surfaces.

Conclusions. It has been established that cyclic temperature variations result in a considerable change in the creep-induced stress redistribution termination time and stress relaxation patterns. However, this change is first determined by cyclic stress variations that arise due to nonuniform cylinder heating along the radius. The account of gradual heating and cooling in the cycle does not lead to an essential change in the stress redistribution patterns and its creep-induced termination time. The temperature dependence of creep constants for the examined steel does not also introduce essential changes in the calculation results, which is probably associated with an average scatter of 
temperature $(80 \mathrm{~K})$ in the cycle and along the radius of the cylinder. However, a wider scatter of temperatures along the radius would cause large thermal stresses in the cylinder, which can end in its fast fracture, even in instantaneous one.

The procedure of refined calculation is sufficiently versatile and can be used in the solution of other problems, substituting asymmetry functions that describe specific cyclic deformation for temperature and stress ones.

The stress distribution in cyclic thermal creep and significant acceleration of its redistribution can greatly influence the estimation of creep rupture strength of engineering structural elements, therefore, it would be necessary to consider them in further calculations.

\section{REFERENCES}

1. V. T. Troshchenko, A. A. Lebedev, V. A. Strizhalo, et al., Mechanical Behavior of Materials under Different Loadings [in Russian], Logos, Kiev (2000).

2. V. V. Krivenyuk, G. A. Okolot, and E. I. Uskov, "Methodological aspects of creep prediction for heat-resistant steels and alloys. Part 2. Prediction over wide ranges of temperatures and loading times," Strength Mater., 39, No. 4, 372-380 (2007).

3. M. V. Borodii, "Life calculations for materials under irregular nonproportional loading," Strength Mater., 39, No. 5, 560-565 (2007).

4. S. N. Shukaev, K. V. Panasovskii, and M. N. Gladskii, "Fatigue life assessment for metal alloys under nonproportional low-cycle loading," Strength Mater., 39, No. 4, 358-364 (2007).

5. J. Lemaitre and J.-L. Chaboche, Mechanics of Solid Materials, University Press, Cambridge (1994).

6. G. S. Pisarenko and N. S. Mozharovskii, Equations and Boundary Problems of the Theory of Plasticity and Creep [in Russian], Naukova Dumka, Kiev (1981).

7. G. S. Pisarenko, N. S. Mozharovskii, and E. A. Antipov, Plasticity and Strength of Materials under Nonstationary Loadings [in Russian], Naukova Dumka, Kiev (1984).

8. V. G. Karnaukhov, I. K. Senchenkov, and B. P. Gumenyuk, Thermomechanical Behavior of Bodies under Harmonic Loading [in Russian], Naukova Dumka, Kiev (1985).

9. G. N. Tret'yachenko and B. S. Karpinos, Strength and Life of Materials under Cyclic Thermal Effects [in Russian], Naukova Dumka, Kiev (1990).

10. G. N. Tret'yachenko, B. S. Karpinos, and V. G. Barilo, Fracture of Materials on Cyclic Heating [in Russian], Naukova Dumka, Kiev (1993).

11. D. V. Breslavskii, O. K. Morachkovskii, and O. A. Uvarova, "Method of asymptotic expansions in the problems of low- and high-cycle creep of materials," Visn. Nats. Tekh. Univ. "KhPI", No. 19, 149-152 (2009).

12. D. V. Breslavskii, O. K. Morachkovskii, and O. A. Tatarinova, "High-temperature creep and long-term strength of structural elements under cyclic loading," Strength Mater., 40, No. 5, 531-537 (2008).

13. O. K. Morachkovskii, "Nonlinear problems of creep of bodies under the action of rapidly oscillating fields," Prikl. Mekh., 28, No. 8, 17-23 (1992).

14. S. Taira and R. Otani, Theory of High-Temperature Strength of Materials [Russian translation from Japanese], Metallurgiya, Moscow (1986).

15. G. Guarnieri, "Characteristics of long-term strength of sheet materials at cyclic loadings and temperatures," in: L. B. Getsov and M. G. Taubina (Eds.), Heat Resisting Alloys at Changing Temperatures and Stresses [in Russian], Gosénergoizdat (1960), pp. 65-110.

16. D. V. Breslavskii, Yu. N. Korytko, and P. N. Lisak, "Software for the finite element simulation of the two-dimensional problems of the creep theory," Visn. Nats. Tekh. Univ. "KhPI", No. 38, $24-29$ (2007).

17. A. M. Sally, Creep of Metals and Heat-Resistant Alloys [Russian translation], Oborongiz, Moscow (1953). 